\title{
Loss of PTEN expression is an independent predictor of favourable survival in endometrial carcinomas
}

\author{
A Akiyama-Abe ${ }^{1}$, T Minaguchi ${ }^{*}{ }^{1}$, Y Nakamura ${ }^{1}, \mathrm{H}_{\text {Michikami }}{ }^{1}$, A Shikama ${ }^{1}, \mathrm{~S}$ Nakao ${ }^{1}, \mathrm{M}$ Sakurai ${ }^{1}, \mathrm{H} \mathrm{Ochi}^{1}$,

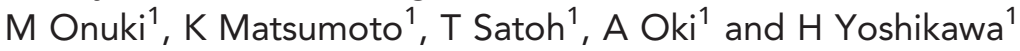 \\ ${ }^{1}$ Department of Obstetrics and Gynecology, Faculty of Medicine, University of Tsukuba, 1-1-1 Tennoudai, Tsukuba, Ibaraki \\ 305-8575, Japan
}

Background: We and others previously reported the prognostic significance of PTEN mutational status on favourable survival in endometrial carcinomas. Here, we demonstrate that loss of PTEN expression in immunohistochemistry is an independent prognostic marker for favourable survival in endometrial carcinomas.

Methods: We conducted immunohistochemical analyses of PTEN, PIK3CA, phosphorylated Akt (p-Akt), and p27 in primary endometrial carcinomas from 221 patients. Mutation of PTEN was analysed further.

Results: Expression of PTEN was lost in 56 patients (25\%), and PIK3CA was overexpressed in 159 patients (72\%). Overexpression of PIK3CA was associated with $p$-Akt overexpression $(P<0.001)$, which was in turn associated with loss of nuclear p27 expression ( $P=0.028)$. Loss of PTEN expression was found to be associated with endometrioid histology $(P=0.03)$, and was inversely associated with the presence of lymphovascular space invasion $(P=0.03)$. Univariate and multivariate survival analyses revealed that factors of PTEN loss, age $<70$, histological grade 1, early International Federation of Gynecology and Obstetrics (FIGO) stage, and absence of lymphovascular invasion were independent prognostic indicators for better overall survival $(P=0.03,0.04,0.01,<0.001$, and 0.03 , respectively). The subset analysis showed a stronger tendency of PTEN loss towards favourable survival in advanced-stage (III and IV) disease than in early-stage (I and II) disease ( $P=0.05$ vs 0.14 ). Moreover, our mutational analysis demonstrated that PTEN expression loss was associated with PTEN-truncating mutations $(P=0.03)$

Conclusion: The current observations further support the prognostic significance of PTEN aberration on favourable outcome in endometrial carcinomas, providing useful implications for the individualised management of the disease.

Dysregulated signalling on the phosphatidylinositol 3' (PI3)kinase/PTEN/Akt cascade is reported to be associated with earlystage disease and favourable prognosis in some types of malignancy, including colon, breast, ovarian, and endometrial cancers. Regarding breast cancer, it has been reported that PIK3CA mutation is associated with positive oestrogen receptor status, low stage, and favourable outcome (Kalinsky et al, 2009). In colorectal cancer, Baba et al (2011) have reported that phosphorylated Akt ( $\mathrm{p}$-Akt) expression is associated with PIK3CA mutation, low stage, and favourable outcome. In ovarian cancer, we and others have recently reported that PIK3CA aberration is associated with favourable survival in ovarian clear cell carcinoma (Rahman et al, 2012; Abe et al, 2013). As for endometrial cancer, we and others previously reported that PTEN mutation is associated with endometrioid histology, early stage, and favourable prognosis (Risinger et al, 1998; Minaguchi et al, 2001). The current study has aimed to investigate the prognostic significance of immunohistochemical (IHC) PTEN expression in endometrial carcinoma. Here, 
we demonstrate that loss of PTEN expression is a significant and independent prognostic factor for favourable survival in the disease. Our observation presents additional evidence for the prognostic significance of PTEN aberration on favourable outcome in endometrial carcinoma, further providing significant implications for the management of the disease including molecular targeted therapies.

\section{MATERIALS AND METHODS}

Patients and specimens. The Ethical Committee of the University of Tsukuba Hospital approved the study protocol. All patients diagnosed with endometrial carcinoma, who were treated in the Department of Obstetrics and Gynecology at the University of Tsukuba Hospital between 1999 and 2009, were identified through our database. A total of 221 patients with endometrial carcinomas were included in the present study, and their medical records were reviewed. A median follow-up duration was 59 months (range, 3-119 months). All patients provided written informed consent. Staging was performed based on the criteria of International Federation of Gynecology and Obstetrics (FIGO). Endometrioid adenocarcinomas were subclassified into three grades $\left(G_{1}, G_{2}\right.$, and $\left.G_{3}\right)$ according to the FIGO criteria. Table 1 summarises the patient characteristics.

Treatment. The operative procedure included hysterectomy, bilateral salpingo-oophorectomy, and systematic aortic and pelvic lymph-node dissection. Radical hysterectomy or semiradical hysterectomy (with removal of vaginal cuff and partial resection of vesico-uterine ligament) was performed on patients with positive findings on cervical stromal invasion by MRI, and simple total hysterectomy was performed on the remainder. Postsurgically, patients with positive peritoneal cytology, adnexal/peritoneal involvement, or pelvic-/aortic-node metastases were treated with combination chemotherapy of paclitaxel and carboplatin. Smallpelvis irradiation (with the lower superior border of field) was indicated for patients with adnexal/peritoneal involvement or deep muscular invasion (more than two-thirds depth in endometrioid $\mathrm{G}_{1,2}$ and more than one-half in $\mathrm{G}_{3}$ or other histotypes). Wholepelvis or periaortic irradiations were administered to pelvic or aortic node-positive patients, respectively.

Immunohistochemistry. Immunohistochemistry was performed as described previously (Abe et al, 2013). Antibodies used were PI3 Kinase p110 $\alpha$ (rabbit monoclonal, 1:200; Cell Signaling, Danvers, MA, USA), Anti-Human PTEN (6H2.1) (mouse monoclonal, 1:100; Cascade, Winchester, MA, USA), Phospho-Akt (Ser473) (rabbit monoclonal, 1:50; Cell Signaling), and Anti-p27 (mouse monoclonal, 1:100; BD Pharmingen, Franklin Lakes, NJ, USA). The corresponding normal endometria or stroma provided an internal positive control, and negative controls without addition of primary antibody showed low background staining.

IHC scoring. For semiquantitative analyses, the IHC staining was scored by multiplying the percentages of positive tumour cells (PP: 0 , no positive cell; $1,<10 \% ; 2,10-50 \%$; and $3,>50 \%$ positive tumour cells) by their prevalent degree of staining (SI: 0 , negative; 1, decreased; 2, equivalent; and 3 , increased staining to the corresponding normal tissue). The IHC scores $(\mathrm{IHS}=\mathrm{PP} \times \mathrm{SI}$ ) range from 0 to 9 . The average value from the scores of two independent observers (AA and TM) blinded for clinicopathological parameters was used as the final value. For the evaluation of PTEN expression, IHS $=0$ was considered as negative. For PIK3CA and p-Akt, IHS $>6$ was evaluated as overexpression. For p27, no staining of tumour cell nuclei was evaluated as nuclear negative. Figure 1 shows examples of IHC staining patterns in normal endometria and endometrial carcinomas. For normal

\begin{tabular}{|c|c|}
\hline Characteristic & Number $n=221$ \\
\hline Median age (range) & $57.0(26-84)$ \\
\hline \multicolumn{2}{|l|}{ FIGO stage } \\
\hline I & 128 \\
\hline la & 22 \\
\hline lb & 76 \\
\hline Ic & 30 \\
\hline II & 26 \\
\hline Illa & 10 \\
\hline Ilb & 16 \\
\hline III & 43 \\
\hline Illa & 20 \\
\hline IIllc & 23 \\
\hline IV & 24 \\
\hline $\mathrm{IVa}$ & 2 \\
\hline $\mathrm{IVb}$ & 22 \\
\hline \multicolumn{2}{|l|}{ Histotype } \\
\hline Endometrioid & 196 \\
\hline $\mathrm{G}_{1}$ & 115 \\
\hline $\mathrm{G}_{2}$ & 56 \\
\hline $\mathrm{G}_{3}$ & 25 \\
\hline Serous & 12 \\
\hline Adenosquamous & 4 \\
\hline Clear cell & 4 \\
\hline Poorly differentiated & 1 \\
\hline Undifferentiated & 1 \\
\hline Mixed epithelial & 3 \\
\hline \multicolumn{2}{|l|}{ Primary treatment } \\
\hline Surgery & 221 \\
\hline Lymphadenectomy & 171 \\
\hline Lymph-node sampling & 21 \\
\hline Chemotherapy & 60 \\
\hline $\mathrm{TC}$ & 55 \\
\hline CAP & 4 \\
\hline Irradiation & 58 \\
\hline
\end{tabular}

control, normal endometria from 15 women were examined, and $>90 \%$ of the specimens were scored as 6 for PTEN, p-Akt, and PIK3CA, and $>85 \%$ were positive for $\mathrm{p} 27$, respectively.

DNA extraction and PTEN mutational analysis. Genomic DNA was extracted from tumour areas of formalin-fixed, paraffinembedded archival tissues with a Dneasy Blood \& Tissue Kit (Qiagen, Valencia, CA, USA) according to the manufacturer's instructions. Mutational analysis for PTEN was performed as previously described. Briefly, aberrant bands revealed by SSCP analysis were excised from the gel, amplified by PCR, purified, and submitted to the Operon Biotechnologies (Tokyo, Japan) for direct sequencing.

Statistical analysis. Differences in proportions were evaluated by the Fisher's exact test. Kaplan-Meier survival curves were calculated and compared statistically using the log-rank test. 
The Cox proportional hazard model was used for the univariate and multivariate analyses.

\section{RESULTS}

Our IHC analyses in 221 endometrial carcinomas showed that PTEN expression was lost in 56 cases (25\%), PIK3CA was overexpressed in 159 (72\%), p-Akt was overexpressed in 189 (86\%), and nuclear p27 expression was lost in 143 (65\%) (Table 2). Moreover, overexpressed PIK3CA was significantly associated with p-Akt overexpression $(P<0.001)$, which in turn significantly correlated with negative nuclear p27 expression $(P=0.03)$ (Table 2). These observations are consistent with the signal transduction mechanism where upregulation of PI3 kinase leads to phosphorylation of Akt, which in turn results in translocation of p27 from nucleus to cytoplasm. This consistency strengthens the validity of our IHC analyses.

We subsequently investigated the relationships between IHC results and clinicopathological parameters (Table 3). Loss of PTEN expression was found to be associated with endometrioid histology $(P=0.03)$, and was inversely associated with the presence of lymphovascular invasion $(P=0.03)$. Negative nuclear p27 expression was associated both with endometrioid histology and with $\mathrm{G}_{1}(P=0.008$ and 0.016 , respectively). Negative PTEN showed trends towards younger age $(P=0.10)$, obesity $(P=0.17)$, and less lymph-node metastases (data not shown).

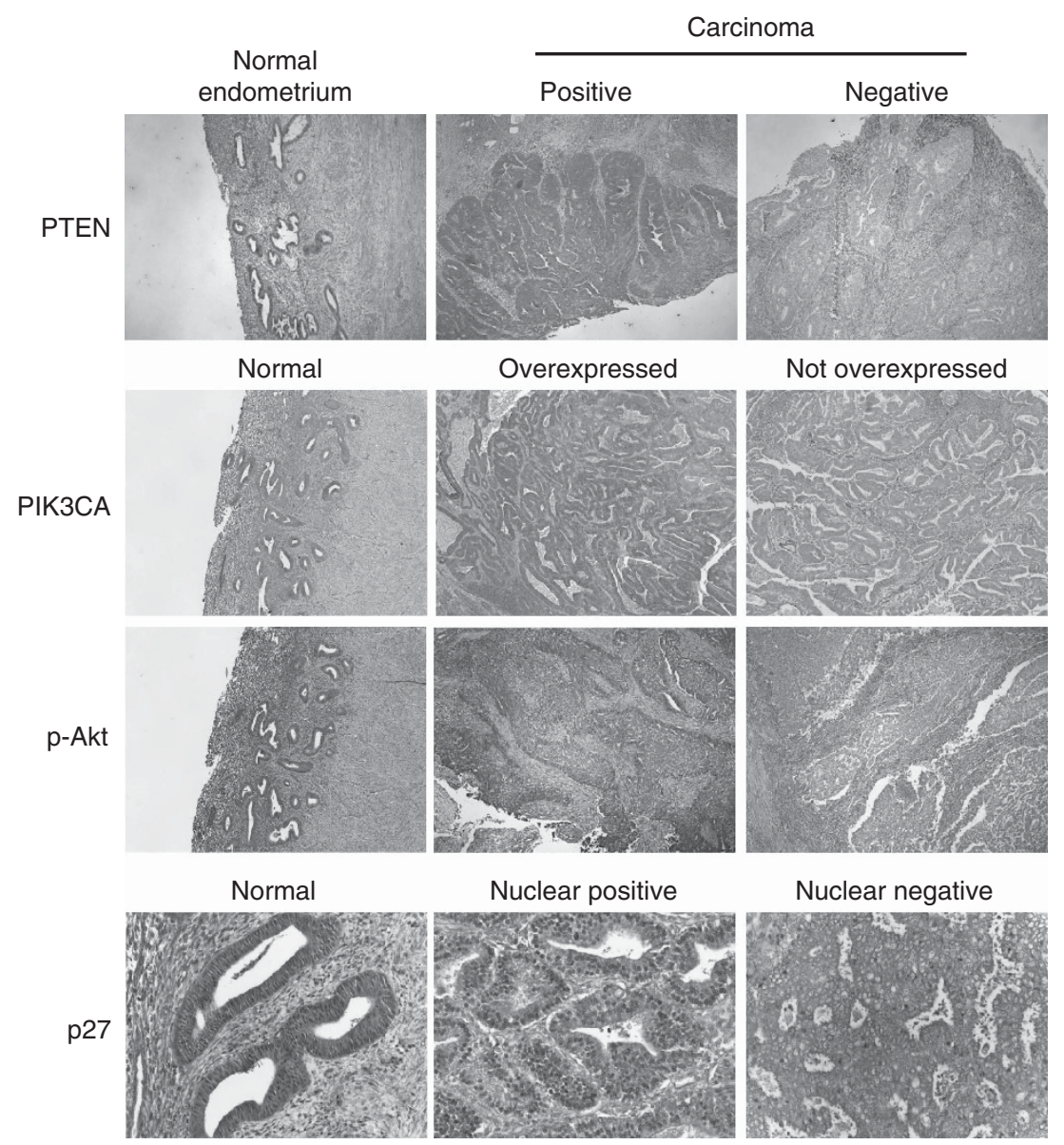

Figure 1. IHC staining patterns of PTEN, PIK3CA, p-Akt, and p27 in normal endometria and endometrial carcinomas. PTEN, PIK3CA, and p-Akt, $\times 100 ;$ p $27, \times 400$

Table 2. Results of IHC evaluation and correlation with p-Akt expression

p-Akt overexpression

\begin{tabular}{l|c|c|c|c|}
\hline Expression & Number, $\mathbf{n}=\mathbf{2 2 1}$ & $(+), \mathbf{n}=\mathbf{1 8 9}$ & $(-), \mathbf{n}=\mathbf{3 2}$ & $\boldsymbol{P}$-value \\
\hline Negative PTEN $(\mathrm{IHS}=0)$ & $56(25 \%)$ & $51(27 \%)$ & $5(16 \%)$ & 0.19 \\
\hline Overexpressed PIK3CA $(\mathrm{IHS}>6)$ & $159(72 \%)$ & $148(78 \%)$ & $11(34 \%)$ & $1.6 \mathrm{E}-06$ \\
\hline Overexpressed p-Akt (IHS >6) & $189(86 \%)$ & - & - & - \\
\hline Negative nuclear p27 (0\%) & $143(65 \%)$ & $128(68 \%)$ & $15(47 \%)$ & 0.03 \\
\hline Abbreviations: IHC=immunohistochemical; IHS = IHC score; p-Akt = phosphorylated Akt.
\end{tabular}




\section{Table 3. Relationship between IHC results and clinicopathological features}

\begin{tabular}{|c|c|c|c|c|c|c|c|c|c|c|c|c|}
\hline \multirow[b]{2}{*}{$\begin{array}{l}\text { Clinicopathological } \\
\text { variables }\end{array}$} & \multicolumn{2}{|c|}{ PTEN expression } & \multicolumn{3}{|c|}{$\begin{array}{c}\text { PIK3CA } \\
\text { overexpression }\end{array}$} & \multicolumn{3}{|c|}{$\begin{array}{c}\text { p-Akt } \\
\text { overexpression }\end{array}$} & \multicolumn{3}{|c|}{$\begin{array}{l}\text { Nuclear p27 } \\
\text { expression }\end{array}$} & \multirow[b]{2}{*}{$\boldsymbol{P}$} \\
\hline & $\begin{array}{c}\text { Negative } \\
n=56\end{array}$ & $\begin{array}{l}\text { Positive } \\
n=165\end{array}$ & $\boldsymbol{P}$ & $\begin{array}{c}(+) \\
n=159\end{array}$ & $\begin{array}{c}(-) \\
n=62\end{array}$ & $\boldsymbol{P}$ & $\begin{array}{c}(+) \\
n=189\end{array}$ & $\begin{array}{c}(-) \\
n=32\end{array}$ & $\boldsymbol{P}$ & $\begin{array}{c}\text { Negative } \\
n=143\end{array}$ & $\begin{array}{c}\text { Positive } \\
n=78\end{array}$ & \\
\hline Age $\geqslant 70$ & $5(9 \%)$ & $31(19 \%)$ & 0.10 & $26(16 \%)$ & $10(16 \%)$ & 1 & $31(16 \%)$ & $5(16 \%)$ & 1 & $25(17 \%)$ & $11(14 \%)$ & 0.57 \\
\hline Pre-menopause & 19 (34\%) & $44(27 \%)$ & 0.31 & $43(27 \%)$ & $20(32 \%)$ & 0.51 & $49(26 \%)$ & 14 (44\%) & 0.055 & 44 (31\%) & 19 (24\%) & 0.35 \\
\hline Null parity & $11(20 \%)$ & $26(16 \%)$ & 0.54 & $25(16 \%)$ & $12(19 \%)$ & 0.55 & $33(17 \%)$ & $4(13 \%)$ & 0.61 & 29 (20\%) & $8(10 \%)$ & 0.061 \\
\hline $\mathrm{BMl}>30$ & $11(20 \%)$ & $19(12 \%)$ & 0.17 & $20(13 \%)$ & $10(16 \%)$ & 0.26 & $24(13 \%)$ & $6(19 \%)$ & 0.40 & $21(15 \%)$ & $9(12 \%)$ & 0.68 \\
\hline DM & $8(14 \%)$ & 31 (48\%) & 0.55 & 25 (16\%) & $14(23 \%)$ & 0.24 & $32(17 \%)$ & $7(22 \%)$ & 0.46 & $25(17 \%)$ & $14(18 \%)$ & 1 \\
\hline $\begin{array}{l}\text { Endometrioid } \\
\text { (vs Non-endometrioid) }\end{array}$ & 53 (98\%) & $143(88 \%)$ & 0.03 & $139(87 \%)$ & 57 (92\%) & 0.48 & $171(90 \%)$ & 25 (78\%) & 0.064 & 133 (93\%) & $63(81 \%)$ & 0.008 \\
\hline $\mathrm{G}_{1}$ & $30(54 \%)$ & $86(52 \%)$ & 0.88 & $84(53 \%)$ & $32(52 \%)$ & 0.88 & $102(54 \%)$ & $14(44 \%)$ & 0.34 & 84 (59\%) & 32 (41\%) & 0.016 \\
\hline $\mathrm{Ml}>1 / 2$ & 17 (30\%) & 64 (39\%) & 0.34 & 55 (35\%) & 26 (42\%) & 0.35 & $68(36 \%)$ & 13 (41\%) & 0.69 & $56(39 \%)$ & 25 (32\%) & 0.31 \\
\hline LVI & $14(25 \%)$ & 70 (42\%) & 0.025 & $58(36 \%)$ & 26 (42\%) & 0.54 & 71 (38\%) & 13 (41\%) & 0.84 & 58 (41\%) & $26(33 \%)$ & 0.31 \\
\hline FIGO stage III/IV & $16(29 \%)$ & $51(31 \%)$ & 0.87 & 46 (29\%) & 21 (34\%) & 0.52 & 57 (30\%) & 10 (31\%) & 1 & 42 (29\%) & 25 (32\%) & 0.76 \\
\hline
\end{tabular}

Next, we compared survival curves according to protein expressions (Figure 2). Patients with loss of PTEN expression showed significantly improved overall survival compared with those without PTEN expression loss (Figure 2A, $P=0.016$ ). In contrast, PIK3CA overexpression, p-Akt overexpression, and negative nuclear $\mathrm{p} 27$ did not show significant differences in overall survival (Figures $2 \mathrm{~B}-\mathrm{D}$ ). When compared in subsets of stage I/II and III/IV, negative PTEN showed more favourable survival in advanced disease than in early disease (Figures 2E and F). Negative PTEN showed trends towards favourable survival, when compared in subsets of both pure endometrioid disease and disease other than pure endometrioid histology (Figures 2G and H).

We further conducted univariate and multivariate analyses of prognostic factors for overall survival. Among various prognostic factors, loss of PTEN expression, age $\geqslant 70, G_{1}$, FIGO stage III/IV, muscular invasion $>1 / 2$, and presence of LVI were found to be significant in the univariate analysis $(P=0.03,<0.001,<0.001$, $<0.001,<0.001$, and $<0.001$, respectively; Table 4 ). Among those significant factors, the following multivariate analysis demonstrated that loss of PTEN expression, age $\geqslant 70, G_{1}$, FIGO stage III/IV, and presence of LVI remained to be significant and independent factors $(P=0.03,0.04,0.01,<0.001$, and 0.03 , respectively; Table 4).

Finally, we performed PTEN mutational analysis using DNAs from 33 archival tumour specimens (Table 5). A total of 27 mutations were detected in 19 samples. Mutations in exon 5 (9) and frameshift and missense mutations (12 each) were most frequent. Loss of PTEN expression $(\mathrm{IHS}=0)$ in immunohistochemistry showed no correlation with the presence of PTEN mutation (67\% vs 52\%, $P=0.49$; Table 6). Interestingly, however, loss of PTEN expression was found to be significantly associated with the presence of frameshift or non-sense mutations, which result in PTEN protein truncation $(67 \%$ vs $24 \%, P=0.03$; Table 6).

\section{DISCUSSION}

Inactivation of the PTEN gene is the most frequent genetic defect in endometrial carcinoma. The most commonly observed PTEN defect is inactivation of both alleles by large deletion and mutation in each allele. Our IHC analyses showed that loss of PTEN expression $(\mathrm{IHS}=0)$ was associated with endometrioid histology and absent lymphovascular invasion (Table 3). This observation suggests that tumours with loss of PTEN expression may have more indolent biological behaviour compared with tumours without PTEN loss. Furthermore, our survival analyses demonstrated that loss of PTEN expression was a significant and independent prognostic predictor for favourable survival in endometrial carcinoma (Table 4), keeping in line with the previously published findings on mostly limited sample size where PTEN mutation is associated with favourable prognosis (Risinger et al, 1998; Minaguchi et al, 2001; Sun et al, 2001; Salvesen et al, 2004). Collectively, our above findings utilising larger sample size suggest that loss of PTEN expression may have prognostic impact on survival through more indolent biological tumour behaviour, further confirming the prognostic significance of PTEN aberration.

Given the tumour suppressor function of PTEN (Minaguchi et al, 1999), one would expect that PTEN inactivation would imply poor prognosis. Endometrial cancers develop through accumulation of multiple genetic and epigenetic aberrations. Some tumours acquire malignant characteristics through PTEN inactivation, while others do so through aberrations of other genes; those aberrations may lead to more aggressive phenotype due to more detrimental molecular events than PTEN inactivation does. Meanwhile, another possible explanation for the impact of PTEN inactivation on good prognosis may be tumour suppressive roles of Akt, the pivotal effector downstream of PTEN (Wyszomierski and $\mathrm{Yu}, 2005)$. Akt reportedly blocks cancer cell mortality and invasion through the transcription factor NFAT (Yoeli-Lerner et al, 2005) or downregulation of RHO activity (Liu et al, 2006). The inhibitory effect of Akt activation on cancer cell cycle has also been reported (Kodama et al, 2002). Another study has indicated that Akt activation can promote tumorigenesis, but suppresses tumour invasion (Hutchinson et al, 2004). In our study, however, neither p-Akt nor p27 did show any prognostic significance for survival, although both proteins showed the expression patterns that are consistent with the signalling pathway. Accordingly, the observed PTEN impact on survival is not likely to be attributed to functions of downstream effectors but rather PTEN genetic aberration itself, 

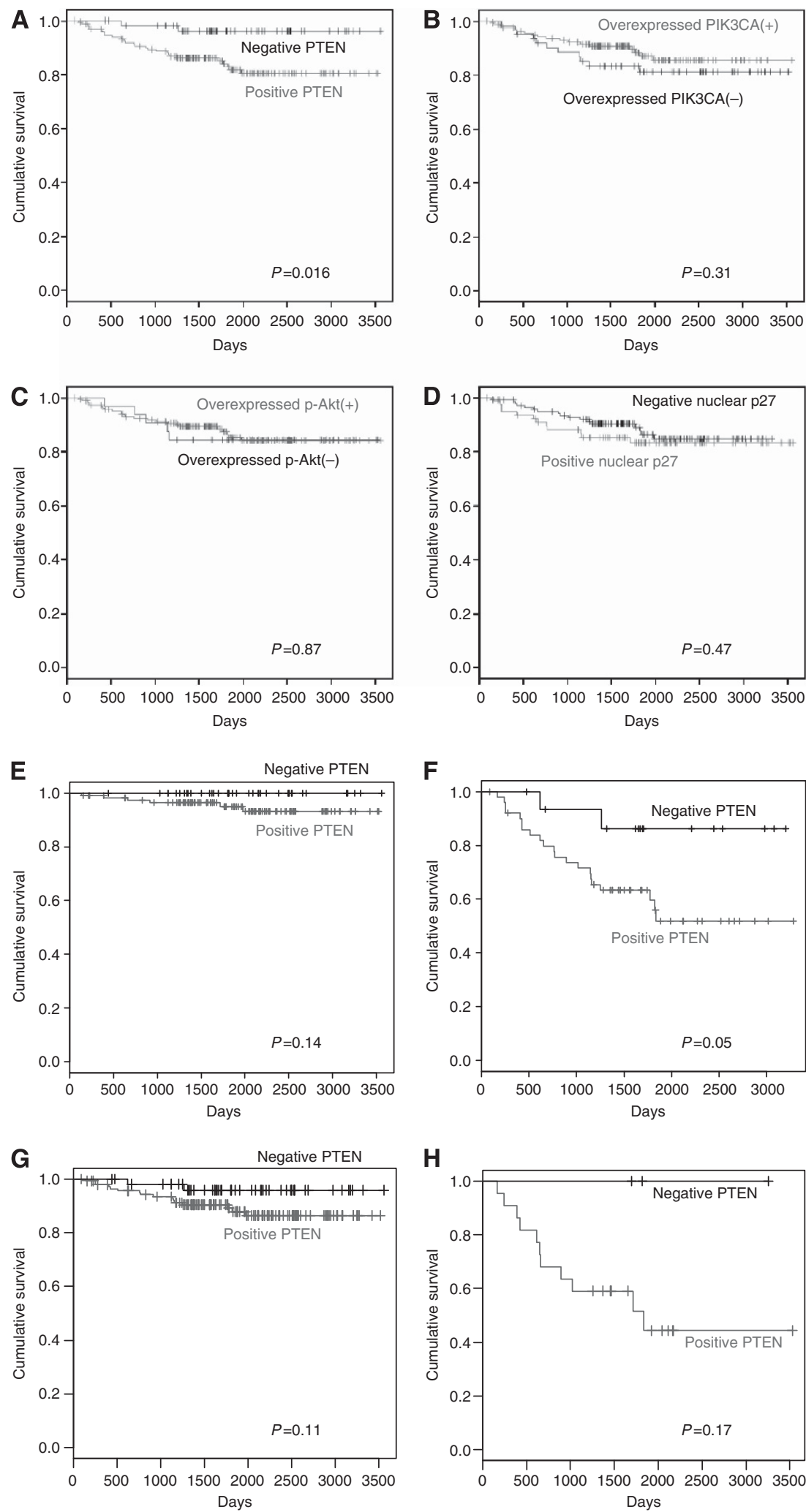

Figure 2. Kaplan-Meier curves for overall survival according to protein expression levels in endometrial carcinomas. (A) Patients with negative PTEN $(n=56)$ vs positive PTEN $(n=165)$; (B) patients with overexpressed PIK3CA $(n=159)$ vs without overexpressed PIK3CA ( $n=62)$; (C) patients with overexpressed p-Akt $(n=189)$ vs without overexpressed p-Akt $(n=32)$; (D) patients with negative nuclear p27 ( $n=143)$ vs positive nuclear p27 ( $n=78)$; $(E)$ patients with negative PTEN $(n=40)$ vs positive PTEN $(n=114)$ in early-stage disease (stages I and II); ( $F)$ patients with negative PTEN $(n=16)$ vs positive PTEN $(n=51)$ in advanced disease (stages III and IV); (G) patients with negative PTEN ( $n=53$ ) vs positive PTEN $(n=143)$ in pure endometrioid disease; $(H)$ patients with negative PTEN $(n=3)$ vs positive PTEN $(n=22)$ in disease other than pure endometrioid histology. 
Table 4. Univariate and multivariate analyses of prognostic factors for overall survival

Univariate

Multivariate

\begin{tabular}{|c|c|c|c|c|c|c|}
\hline Prognostic factor & HR & $95 \% \mathrm{Cl}$ & $\boldsymbol{P}$-value & HR & $95 \% \mathrm{Cl}$ & $\boldsymbol{P}$-value \\
\hline PTEN negative (vs positive) & 0.20 & $0.05-0.86$ & 0.03 & 0.21 & $0.05-0.88$ & 0.03 \\
\hline PIK3CA overexpression (vs remainder) & 0.68 & $0.32-1.43$ & 0.31 & - & - & - \\
\hline $\mathrm{p}$-Akt overexpression (vs remainder) & 0.92 & $0.35-2.42$ & 0.87 & - & - & - \\
\hline Nuclear p27 negative (vs positive) & 0.76 & $0.37-1.60$ & 0.47 & - & - & - \\
\hline Age $\geqslant 70(v s<70)$ & 3.67 & $1.69-7.98$ & $<0.001$ & 2.44 & $1.06-5.63$ & 0.04 \\
\hline $\mathrm{G}_{1}$ (vs $\mathrm{G}_{2-3}$ /non-endometrioid) & 0.16 & $0.06-0.42$ & $<0.001$ & 0.28 & $0.11-0.76$ & 0.01 \\
\hline FIGO stage III/IV (vs I/II) & 10.9 & $4.44-26.9$ & $<0.001$ & 5.70 & $2.17-15.0$ & $<0.001$ \\
\hline $\mathrm{Ml}>1 / 2(v s \leqslant 1 / 2)$ & 9.50 & $3.62-24.9$ & $<0.001$ & 2.24 & $0.76-6.60$ & 0.14 \\
\hline LVI present (vs absent) & 7.42 & $3.02-18.2$ & $<0.001$ & 3.01 & $1.14-7.95$ & 0.03 \\
\hline
\end{tabular}

Abbreviations: $\mathrm{Cl}=$ confidence interval; $\mathrm{FIGO}=$ International Federation of Gynecology and Obstetrics; $\mathrm{HR}=$ hazard ratio; $\mathrm{LVI}=$ lymphovascular invasion; $\mathrm{MI}=$ muscular invasion; $\mathrm{p}-\mathrm{Akt}=$ phosphorylated Akt.

Table 5. PTEN mutational status and IHC score in endometrial carcinoma cases

\begin{tabular}{|c|c|c|c|c|c|c|c|c|c|c|c|c|c|c|}
\hline Case & Histotype & $\begin{array}{c}\text { Mutated } \\
\text { exons }\end{array}$ & $\mathrm{NCL}$ & AA & $\mathrm{NCL}$ & AA & $\mathrm{NCL}$ & AA & $\mathrm{NCL}$ & AA & Mut & $\begin{array}{c}\text { Mut only } \\
\text { outside } \\
\text { exons 5-7 }\end{array}$ & $\begin{array}{c}\text { PTEN } \\
\text { truncation }\end{array}$ & IHS \\
\hline 1 & $\mathrm{G}_{2}$ & 8 & 963_964insA & $\mathrm{T} 321 \mathrm{fs} * 3$ & & & & & & & 1 & 1 & 1 & 0 \\
\hline 2 & $\mathrm{G}_{1}$ & 8 & $1008 C>A$ & Y336* & & & & & & & 1 & 1 & 1 & 0 \\
\hline 3 & $\mathrm{G}_{2}$ & & & & & & & & & & 0 & 0 & 0 & 4.5 \\
\hline 4 & Mixed & & & & & & & & & & 0 & 0 & 0 & 6 \\
\hline 5 & $\mathrm{G}_{1}$ & & & & & & & & & & 0 & 0 & 0 & 6 \\
\hline 6 & Mixed & & & & & & & & & & 0 & 0 & 0 & 0 \\
\hline 7 & $\mathrm{G}_{2}$ & & & & & & & & & & 0 & 0 & 0 & 6 \\
\hline 8 & $\mathrm{G}_{1}$ & 5 & $389 \mathrm{G}>\mathrm{C}$ & R130P & & & & & & & 1 & 0 & 0 & 6 \\
\hline 9 & $\mathrm{G}_{1}$ & 5 & $388 C>G$ & R130G & & & & & & & 1 & 0 & 0 & 6 \\
\hline 10 & $\mathrm{G}_{1}$ & 8 & $907 \mathrm{del} A$ & $1303 \mathrm{fs}^{\star 4} 4$ & & & & & & & 1 & 1 & 1 & 0 \\
\hline 11 & $\mathrm{G}_{3}$ & 7 & 800delA & $\mathrm{K} 267 \mathrm{fs}^{*} \mathrm{9}$ & & & & & & & 1 & 0 & 1 & 6 \\
\hline 12 & Clear cell & & & & & & & & & & 0 & 0 & 0 & 6 \\
\hline 13 & $\mathrm{G}_{1}$ & & & & & & & & & & 0 & 0 & 0 & 0 \\
\hline 14 & $\mathrm{G}_{2}$ & 5,8 & $388 C>G$ & R130G & $962 \mathrm{C}>\mathrm{AT}$ & T321 $1 \mathrm{fs}^{\star} 4$ & & & & & 1 & 0 & 1 & 2 \\
\hline 15 & $\mathrm{G}_{1}$ & & & & & & & & & & 0 & 0 & 0 & 0 \\
\hline 16 & $\mathrm{G}_{3}$ & $3,5,6,6$ & $208 C>A$ & L70I & $431 \mathrm{~A}>\mathrm{C}$ & K144Y & $517 \mathrm{C}>\mathrm{T}$ & R173C & $601 \mathrm{G}>\mathrm{T}$ & E201* & 1 & 0 & 1 & 0 \\
\hline 17 & $\mathrm{G}_{2}$ & 7 & $640 \_655>A C T$ & Q214fs*3 & & & & & & & 1 & 0 & 1 & 0 \\
\hline 18 & $\mathrm{G}_{1}$ & 5 & $389 \mathrm{G}>\mathrm{A}$ & R1300 & & & & & & & 1 & 0 & 0 & 4 \\
\hline 19 & $\mathrm{G}_{2}$ & 5,9 & 405_406delAT & $1135 f s^{\star} 44$ & $1028 \mathrm{TT}>\mathrm{G}$ & V343G & & & & & 1 & 0 & 1 & 0 \\
\hline 20 & $\mathrm{G}_{1}$ & 1 & 64_70del7bp & $\mathrm{D} 22 \mathrm{fs} * 2$ & & & & & & & 1 & 1 & 1 & 0 \\
\hline 21 & $\mathrm{G}_{1}$ & 7 & 710delA & $\mathrm{K} 237 \mathrm{fs}^{\star 1} 19$ & & & & & & & 1 & 0 & 1 & 0 \\
\hline 22 & $\mathrm{G}_{1}$ & 2 & $80 \mathrm{G}>\mathrm{A}$ & Y27C & & & & & & & 1 & 1 & 0 & 3 \\
\hline 23 & Mixed & & & & & & & & & & 0 & 0 & 0 & 3 \\
\hline 24 & $\mathrm{G}_{2}$ & & & & & & & & & & 0 & 0 & 0 & 3 \\
\hline 25 & Clear cell & 1 & 38_39insC & $\mathrm{K} 13 \mathrm{fs}^{*} * 30$ & & & & & & & 1 & 1 & 1 & 6 \\
\hline 26 & $\mathrm{G}_{1}$ & 5,6 & 440_441insA & $\mathrm{K} 147 \mathrm{fs} * 32$ & $562 \_576>C$ & Y188fs $* 8$ & & & & & 1 & 0 & 1 & 3 \\
\hline 27 & $\mathrm{G}_{1}$ & 2 & $103 A>G$ & M35V & & & & & & & 1 & 1 & 0 & 6 \\
\hline 28 & $\mathrm{G}_{1}$ & & & & & & & & & & 0 & 0 & 0 & 9 \\
\hline 29 & $\mathrm{G}_{1}$ & 5,5 & $263 \mathrm{~A}>\mathrm{G}$ & Y88C & $276 C>G$ & D92E & & & & & 1 & 0 & 0 & 9 \\
\hline 30 & $\mathrm{G}_{2}$ & & & & & & & & & & 0 & 0 & 0 & 0 \\
\hline 31 & $\mathrm{G}_{2}$ & & & & & & & & & & 0 & 0 & 0 & 9 \\
\hline 32 & $\mathrm{G}_{1}$ & & & & & & & & & & 0 & 0 & 0 & 3 \\
\hline 33 & $\mathrm{G}_{1}$ & 6,7 & 611delC & $\mathrm{P} 204 \mathrm{fs}^{\star} 17$ & $796 \mathrm{~A}>\mathrm{T}$ & K266* & & & & & 1 & 0 & 1 & 3 \\
\hline
\end{tabular}


Table 6. Relationship between PTEN mutational status and IHC results

\begin{tabular}{|c|c|c|c|c|c|c|}
\hline PTEN IHC & Mutation in any exon & $\boldsymbol{P}$-value & $\begin{array}{l}\text { Mutation only outside } \\
\text { exons } 5-7\end{array}$ & $\boldsymbol{P}$-value & PTEN truncation & $\boldsymbol{P}$-value \\
\hline $\mathrm{IHS}=0$ & $8 / 12(67 \%)$ & 0.49 & $4 / 12$ (33\%) & 0.38 & $8 / 12(67 \%)$ & 0.03 \\
\hline $\mathrm{HIS}>0$ & $11 / 21(52 \%)$ & & $3 / 21(14 \%)$ & & $5 / 21(24 \%)$ & \\
\hline $\mathrm{IHS}<6$ & $13 / 21(62 \%)$ & 0.72 & $5 / 21(24 \%)$ & 1.0 & $11 / 21(52 \%)$ & 0.07 \\
\hline $\mathrm{HIS} \geqslant 6$ & $6 / 12(50 \%)$ & & $2 / 12(17 \%)$ & & $2 / 12(17 \%)$ & \\
\hline
\end{tabular}

Abbreviations: $\mathrm{IHC}=$ immunohistochemical; $\mathrm{IHS}=\mathrm{IHC}$ score.

which may represent the biological and clinical characteristics of tumour.

The antibody used for our PTEN immunohistochemistry, that is, 6H2.1, recognises the C-terminal 100 amino acids of PTEN protein. Theoretically, not all mutations of PTEN may be detected as altered protein expression. Frameshift and non-sense mutations that result in truncating the C-terminal 100 amino acids of PTEN should be observed as null staining, while missense mutations may not be recognised by altered staining. In fact, our mutational analysis demonstrated that loss of PTEN expression was not associated with PTEN mutational status, but rather with the presence of frameshift or non-sense mutations that produce truncated PTEN proteins (Table 6). It can be speculated that PTEN-truncating mutations may spare functionally important regions of PTEN protein, leading to better outcome, compared with tumours with other PTEN mutations and mutations of other genes. Indeed, we previously reported that PTEN mutation only outside exons 5-7 was an independent prognostic factor for favourable survival in endometrial carcinoma, possibly due to incomplete disruption of protein function by sparing functionally important elements located inside exons 5-7 (Minaguchi et al, 2001). However, the current study failed to find statistically significant correlation between loss of PTEN expression and mutations only outside exons 5-7 (Table 6). This discordance may be due to small sample size for mutational analysis in the current study. In any case, together with the published findings, our results further support the prognostic significance of PTEN aberration on favourable prognosis in endometrial carcinoma. Future studies are warranted to verify our observations as well as to elucidate mechanisms whereby PTEN inactivation affects tumour behaviour and patient prognosis.

Recently, various molecular agents targeting the PI3-kinase/ PTEN/Akt pathway have been developed including mTOR inhibitors such as everolimus and temsirolimus, and numerous clinical studies are ongoing in endometrial cancer (Oza et al, 2011). Today, it is getting important in terms of health economics to identify biomarkers predicting the sensitivity of tumour to those expensive molecular agents. As our subset analysis showed a stronger trend of PTEN loss towards favourable survival in advanced-stage disease than in early-stage disease, patients with tumour negative for PTEN protein expression may benefit more from the therapeutics targeting molecules downstream of PTEN particularly in patients with advanced-stage diseases.

In conclusion, we have demonstrated here that loss of PTEN expression is a significant and independent predictor for favourable survival in endometrial carcinomas. The current data suggest that favourable outcome of tumours with PTEN expression loss may be due to more indolent biological behaviour of tumour. Furthermore, loss of PTEN expression was found to be associated with PTEN-truncating mutations. Our observations provide significant implications for the individualised management of endometrial cancer including the use of molecular targeted agents.

\section{ACKNOWLEDGEMENTS}

This study was partially supported by the Grant-in-Aid for Scientific Research (No. 24592503) from the Ministry of Education, Culture, Sports, Science, and Technology, Tokyo, Japan.

\section{CONFLICT OF INTEREST}

The authors declare no conflict of interest.

\section{REFERENCES}

Abe A, Minaguchi T, Ochi H, Onuki M, Okada S, Matsumoto K, Satoh T, Oki A, Yoshikawa H (2013) PIK3CA overexpression is a possible prognostic factor for favorable survival in ovarian clear cell carcinoma. Hum Pathol 44(2): 199-207.

Baba Y, Nosho K, Shima K, Hayashi M, Meyerhardt JA, Chan AT, Giovannucci E, Fuchs CS, Ogino S (2011) Phosphorylated AKT expression is associated with PIK3CA mutation, low stage, and favorable outcome in 717 colorectal cancers. Cancer 117(7): 1399-1408.

Hutchinson JN, Jin J, Cardiff RD, Woodgett JR, Muller WJ (2004) Activation of Akt-1 (PKB-alpha) can accelerate ErbB-2-mediated mammary tumorigenesis but suppresses tumor invasion. Cancer Res 64(9): 3171-3178.

Kalinsky K, Jacks LM, Heguy A, Patil S, Drobnjak M, Bhanot UK, Hedvat CV, Traina TA, Solit D, Gerald W, Moynahan ME (2009) PIK3CA mutation associates with improved outcome in breast cancer. Clin Cancer Res 15(16): 5049-5059.

Kodama Y, Baxter RC, Martin JL (2002) Insulin-like growth factor-I inhibits cell growth in the a549 non-small lung cancer cell line. Am J Respir Cell Mol Biol 27(3): 336-344.

Liu H, Radisky DC, Nelson CM, Zhang H, Fata JE, Roth RA, Bissell MJ (2006) Mechanism of Akt1 inhibition of breast cancer cell invasion reveals a protumorigenic role for TSC2. Proc Natl Acad Sci USA 103(11): 4134-4139.

Minaguchi T, Mori T, Kanamori Y, Matsushima M, Yoshikawa H, Taketani Y, Nakamura Y (1999) Growth suppression of human ovarian cancer cells by adenovirus-mediated transfer of the PTEN gene. Cancer Res 59(24): 6063-6067.

Minaguchi T, Yoshikawa H, Oda K, Ishino T, Yasugi T, Onda T, Nakagawa S, Matsumoto K, Kawana K, Taketani Y (2001) PTEN mutation located only outside exons 5, 6, and 7 is an independent predictor of favorable survival in endometrial carcinomas. Clin Cancer Res 7(9): 2636-2642.

Oza AM, Elit L, Tsao MS, Kamel-Reid S, Biagi J, Provencher DM, Gotlieb WH, Hoskins PJ, Ghatage P, Tonkin KS, Mackay HJ, Mazurka J, Sederias J, Ivy P, Dancey JE, Eisenhauer EA (2011) Phase II study of temsirolimus in women with recurrent or metastatic endometrial cancer: a trial of the NCIC Clinical Trials Group. J Clin Oncol 29(24): 3278-3285.

Rahman M, Nakayama K, Rahman MT, Nakayama N, Ishikawa M, Katagiri A, Iida K, Nakayama S, Otsuki Y, Shih IeM, Miyazaki K (2012) Clinicopathologic and biological analysis of PIK3CA mutation in ovarian clear cell carcinoma. Hum Pathol 43(12): 2197-2206. 
Risinger JI, Hayes K, Maxwell GL, Carney ME, Dodge RK, Barrett JC, Berchuck A (1998) PTEN mutation in endometrial cancers is associated with favorable clinical and pathologic characteristics. Clin Cancer Res 4(12): 3005-3010.

Salvesen HB, Stefansson I, Kretzschmar EI, Gruber P, MacDonald ND, Ryan A, Jacobs IJ, Akslen LA, Das S (2004) Significance of PTEN alterations in endometrial carcinoma: a population-based study of mutations, promoter methylation and PTEN protein expression. Int J Oncol 25(6): 1615-1623.

Sun H, Enomoto T, Fujita M, Wada H, Yoshino K, Ozaki K, Nakamura T, Murata Y (2001) Mutational analysis of the PTEN gene in endometrial carcinoma and hyperplasia. Am J Clin Pathol 115(1): 32-38.
Wyszomierski SL, Yu D (2005) A knotty turnabout?: Akt1 as a metastasis suppressor. Cancer Cell 8(6): 437-439.

Yoeli-Lerner M, Yiu GK, Rabinovitz I, Erhardt P, Jauliac S, Toker A (2005) Akt blocks breast cancer cell motility and invasion through the transcription factor NFAT. Mol Cell 20(4): 539-550.

This work is published under the standard license to publish agreement. After 12 months the work will become freely available and the license terms will switch to a Creative Commons AttributionNonCommercial-Share Alike 3.0 Unported License. 